PROCEEDINGS OF THE

AMERICAN MATHEMATICAL SOCIETY

Volume 139, Number 12, December 2011, Pages 4163-4171

S 0002-9939(2011)10844-X

Article electronically published on April 12, 2011

\title{
IMPLICATIONS OF THE HASSE PRINCIPLE \\ FOR ZERO CYCLES OF DEGREE ONE ON PRINCIPAL HOMOGENEOUS SPACES
}

\author{
JODI BLACK \\ (Communicated by Ken Ono)
}

\begin{abstract}
Let $k$ be a perfect field of virtual cohomological dimension $\leq 2$. Let $G$ be a connected linear algebraic group over $k$ such that $G^{s c}$ satisfies a Hasse principle over $k$. Let $X$ be a principal homogeneous space under $G$ over $k$. We show that if $X$ admits a zero cycle of degree one, then $X$ has a $k$-rational point.
\end{abstract}

\section{INTRODUCTION}

The following question of Serre [10, p. 192] is open in general.

Q: Let $k$ be a field and $G$ a connected linear algebraic group defined over $k$. Let $X$ be a principal homogeneous space under $G$ over $k$. Suppose $X$ admits a zero cycle of degree one. Does $X$ have a $k$-rational point?

Let $k$ be a number field, let $V$ be the set of places of $k$ and let $k_{v}$ denote the completion of $k$ at a place $v$. We say that a connected linear algebraic group $G$ defined over $k$ satisfies a Hasse principle over $k$ if the map $H^{1}(k, G) \rightarrow \prod_{v \in V} H^{1}\left(k_{v}, G\right)$ is injective. Let $V_{r}$ denote the set of real places of $k$. If $G$ is simply connected, then by a theorem of Kneser, the Hasse principle reduces to injectivity of the maps $H^{1}(k, G) \rightarrow \prod_{v \in V_{r}} H^{1}\left(k_{v}, G\right)$. That this result holds is a theorem due to Kneser, Harder and Chernousov [3], 4, [5]. Sansuc used this Hasse principle to show that $\mathbf{Q}$ has a positive answer for number fields.

Let $k$ be any field and $\Omega$ the set of orderings of $k$. For $v \in \Omega$ let $k_{v}$ denote the real closure of $k$ at $v$. We say that a connected linear algebraic group $G$ defined over $k$ satisfies a Hasse principle over $k$ if the map $H^{1}(k, G) \rightarrow \prod_{v \in \Omega} H^{1}\left(k_{v}, G\right)$ is injective. It is a conjecture of Colliot-Thélène [1, p. 652] that a simply connected semisimple group satisfies a Hasse principle over a perfect field of virtual cohomological dimension $\leq 2$. Bayer and Parimala 1 have given a proof in the case where $G$ is of classical type, type $F_{4}$ and type $G_{2}$.

Our goal in this paper is to extend Sansuc's result by providing a positive answer to $\mathbf{Q}$ when $k$ is a perfect field of virtual cohomological dimension $\leq 2$ and $G^{s c}$ satisfies a Hasse principle over $k$. More precisely, we prove the following:

Received by the editors October 7, 2010

2010 Mathematics Subject Classification. Primary 11E72; Secondary 11E57.

The results in this work are from a doctoral dissertation in progress under the direction of R. Parimala, whom the author sincerely thanks for her guidance.

(C)2011 American Mathematical Society Reverts to public domain 28 years from publication 
Theorem 0.1. Let $k$ be a perfect field of virtual cohomological dimension $\leq 2$. Let $\left\{L_{i}\right\}_{1 \leq i \leq m}$ be a set of finite field extensions of $k$ such that the greatest common divisor of the degrees of the extensions $\left[L_{i}: k\right]$ is 1 . Let $G$ be a connected linear algebraic group over $k$. If $G^{s c}$ satisfies a Hasse principle over $k$, then the canonical map

$$
H^{1}(k, G) \rightarrow \prod_{i=1}^{m} H^{1}\left(L_{i}, G\right)
$$

has trivial kernel.

We obtain the following as a corollary:

Corollary 0.2. Let $k$ be a perfect field of virtual cohomological dimension $\leq 2$. Let $\left\{L_{i}\right\}_{1 \leq i \leq m}$ be a set of finite field extensions of $k$ such that the greatest common divisor of the degrees of the extensions $\left[L_{i}: k\right]$ is 1 . Let $G$ be a connected linear algebraic group over $k$. If the simple factors of $G^{\text {sc }}$ are of classical type, type $F_{4}$ or type $G_{2}$, then the canonical map

$$
H^{1}(k, G) \rightarrow \prod_{i=1}^{m} H^{1}\left(L_{i}, G\right)
$$

is injective.

Sansuc's proof of a positive answer to $\mathbf{Q}$ over number fields relies on the surjectivity of the map $H^{1}(k, \mu) \rightarrow \prod_{v \in V_{r}} H^{1}\left(k_{v}, \mu\right)$ for $\mu$ a finite commutative group scheme. This result is a consequence of the Chebotarev density theorem and does not extend to a general field of virtual cohomological dimension $\leq 2$. Even in the case $\mu=\mu_{2}$, the surjectivity of the map $H^{1}(k, \mu) \rightarrow \prod_{v \in \Omega} H^{1}\left(k_{v}, \mu\right)$ imposes severe conditions on $k$ such as the SAP property. The main content of this paper is to replace the arithmetic in Sansuc's paper with a norm principle over a real closed field.

\section{Algebraic groups}

In this section, we review some well-known facts from the theory of algebraic groups and define some notation used in the remainder of the work.

Let $k$ be a field. An algebraic group $G$ over $k$ is a smooth group scheme of finite type. A surjective morphism of algebraic groups with finite kernel is called an isogeny of algebraic groups. An isogeny $G_{1} \rightarrow G_{2}$ is said to be central if its kernel is a central subgroup of $G_{1}$.

An algebraic torus is an algebraic group $T$ such that $T(\bar{k})$ is isomorphic to a product of multiplicative groups $G_{m, \bar{k}}$. A torus $T$ is said to be quasitrivial if it is a product of groups of the form $R_{E_{i} / k} G_{m}$, where $\left\{E_{i}\right\}_{1 \leq i \leq r}$ is a family of finite field extensions of $k$.

An algebraic group $G$ is called linear if it is isomorphic to a closed subgroup of $G L_{n}$ for some $n$, or equivalently, if its underlying algebraic variety is affine. Of particular interest among connected linear algebraic groups are semisimple groups and reductive groups.

A connected linear algebraic group is called semisimple if it has no nontrivial, connected, solvable, normal subgroups. A semisimple group $G$ is said to be simply connected if every central isogeny $G^{\prime} \rightarrow G$ is an isomorphism. We can associate to any semisimple group a simply connected group $\tilde{G}$ (unique up to isomorphism) 
such that there is a central isogeny $\tilde{G} \rightarrow G$. We refer to $\tilde{G}$ as the simply connected cover of $G$.

Any simply connected semisimple group is a product of simply connected simple algebraic groups [6, Theorem 26.8]. Any simple algebraic group belongs to one of four infinite families $A_{n}, B_{n}, C_{n}, D_{n}$ or is of type $E_{6}, E_{7}, E_{8}, F_{4}$ or $G_{2}$ (see for example [6, §26]). A simple group which is of type $A_{n}, B_{n}, C_{n}$ or $D_{n}$ but not of type trialitarian $D_{4}$ is said to be a classical group. All other simple groups are called exceptional groups.

A connected linear algebraic group is called reductive if it has no nontrivial, connected, unipotent, normal subgroups. Given a connected linear algebraic group $G$, the unipotent radical of $G$, denoted $G^{u}$, is the maximal connected unipotent normal subgroup of $G$. It is clear that $G / G^{u}$ is always a reductive group. We denote $G / G^{u}$ by $G^{\text {red }}$. The commutator subgroup of $G^{\text {red }}$ is a semisimple group which we denote $G^{s s}$. We denote the simply connected cover of $G^{s s}$ by $G^{s c}$.

A special covering of a reductive group $G$ is an isogeny

$$
1 \rightarrow \mu \rightarrow G_{0} \times S \rightarrow G \rightarrow 1,
$$

where $G_{0}$ is a simply connected semisimple algebraic $k$-group and $S$ is a quasitrival $k$-torus. Given a reductive group $G$ there exists an integer $n$ and a quasitrival torus $T$ such that $G^{n} \times T$ admits a special covering [8, Lemme 1.10].

\section{Galois Cohomology and zero CyCles}

For our convenience, we will discuss $\mathbf{Q}$ in the context of Galois Cohomology. We briefly review some of the notions from Galois Cohomology that we will use and then restate $\mathbf{Q}$ in this setting.

Let $k$ be a field and $\Gamma_{k}=\operatorname{Gal}(\bar{k} / k)$ be the absolute Galois group of $k$. For an algebraic $k$-group $G$, let $H^{i}(k, G)=H^{i}\left(\Gamma_{k}, G(\bar{k})\right)$ denote the Galois Cohomology of $G$ with the assumption $i \leq 1$ if $G$ is not abelian. For any $k$-group $G, H^{0}(k, G)=$ $G(k)$ and $H^{1}(k, G)$ is a pointed set which classifies the isomorphism classes of principal homogeneous spaces under $G$ over $k$. The point in $H^{1}(k, G)$ corresponds to the principal homogeneous space with rational point. We will interchangeably denote the point in $H^{1}(k, G)$ by point or 1 .

Each $\Gamma_{k}$-homomorphism $f: G \rightarrow G^{\prime}$ induces a functorial map $H^{i}(k, G) \rightarrow$ $H^{i}\left(k, G^{\prime}\right)$, which we shall also denote by $f$. Given an exact sequence of $k$-groups,

$$
1 \longrightarrow G_{1} \stackrel{f_{1}}{\longrightarrow} G_{2} \stackrel{f_{2}}{\longrightarrow} G_{3} \rightarrow 1
$$

there exists a connecting map $\delta_{0}: G_{3}(k) \rightarrow H^{1}\left(k, G_{1}\right)$ such that the following is an exact sequence of pointed sets:

$$
G_{1}(k) \stackrel{f_{1}}{\longrightarrow} G_{2}(k) \stackrel{f_{2}}{\longrightarrow} G_{3}(k) \stackrel{\delta_{0}}{\longrightarrow} H^{1}\left(k, G_{1}\right) \stackrel{f_{1}}{\longrightarrow} H^{1}\left(k, G_{2}\right) \stackrel{f_{2}}{\longrightarrow} H^{1}\left(k, G_{3}\right) .
$$

If $G_{1}$ is central in $G_{2}$, there is in addition a connecting map $\delta_{1}: H^{1}\left(k, G_{3}\right) \rightarrow$ $H^{2}\left(k, G_{1}\right)$ such that the following is an exact sequence of pointed sets:

$$
G_{3}(k) \stackrel{\delta_{0}}{\longrightarrow} H^{1}\left(k, G_{1}\right) \stackrel{f_{1}}{\longrightarrow} H^{1}\left(k, G_{2}\right) \stackrel{f_{2}}{\longrightarrow} H^{1}\left(k, G_{3}\right) \stackrel{\delta_{1}}{\longrightarrow} H^{2}\left(k, G_{1}\right) .
$$

Given a field extension $L$ of $k, \operatorname{Gal}(\bar{k} / L) \subset \operatorname{Gal}(\bar{k} / k)$ and there is a restriction homomorphism res : $H^{1}(k, G) \rightarrow H^{1}(L, G)$. If $G$ is a commutative group and if the degree of $L$ over $k$ is finite, there is also a corestriction homomorphism 
cores : $H^{1}(L, G) \rightarrow H^{1}(k, G)$. The composition cores $\circ$ res is multiplication by the degree of $L$ over $k$.

Let $p$ be any prime number. The $p$-cohomological dimension of $k$ is less than or equal to $r$ (written $\left.\operatorname{cd}_{p}(k) \leq r\right)$ if $H^{n}(k, A)=0$ for every $p$-primary torsion $\Gamma_{k^{-}}$ module $A$ and $n>r$. The cohomological dimension of $k$ is less than or equal to $r$ (written $\operatorname{cd}(k) \leq r$ ) if $\operatorname{cd}_{p}(k) \leq r$ for all primes $p$. Finally, the virtual cohomological dimension of $k$, written $\operatorname{vcd}(k)$ is precisely the cohomological dimension of $k(\sqrt{-1})$. If $k$ is a field of positive characteristic, then $\operatorname{vcd}(k)=\operatorname{cd}(k)$.

Let $X$ be a scheme. For any closed point $x \in X$, let $\mathcal{O}_{x}$ be the local ring at $x$ and let $\mathfrak{M}_{x}$ be its maximal ideal. The residue field of $x$, written $k(x)$, is $\mathcal{O}_{x} / \mathfrak{M}_{x}$. Zero cycles of $X$ are elements of the free abelian group on closed points $x \in X$. We may associate to any zero cycle $\sum n_{i} x_{i}$ on $X$ its degree $\sum n_{i}\left[k\left(x_{i}\right): k\right]$, where $k\left(x_{i}\right)$ is the residue field of $x_{i}$.

A closed point with residue field $k$ is called a rational point. It is clear that if $x$ is a closed point of a variety $X$ over $k$, then it is a rational point of $X_{k(x)}$. We have seen that the point in $H^{1}(*, G)$ is the principal homogeneous space under $G$ over * with a rational point. Therefore, a principal homogeneous space $X$ under $G$ over $k$, with zero cycle $\sum n_{i} x_{i}$, is an element of the kernel of the product of the restriction maps $H^{1}(k, G) \rightarrow \prod H^{1}\left(k\left(x_{i}\right), G\right)$. If the zero cycle is of degree one, then the field extensions $k\left(x_{i}\right)$ are necessarily of coprime degree over $k$.

Guided by this insight, one may restate $\mathbf{Q}$ as follows.

Q: Let $k$ be a field and let $G$ be a connected, linear algebraic group defined over $k$. Let $\left\{L_{i}\right\}_{1 \leq i \leq m}$ be a collection of finite extensions of $k$ with $\operatorname{gcd}\left(\left[L_{i}\right.\right.$ : $k])=1$. Does the canonical map

$$
H^{1}(k, G) \rightarrow \prod_{i=1}^{m} H^{1}\left(L_{i}, G\right)
$$

have trivial kernel?

\section{ORDERINGS OF A FIELD}

We recall some basic properties of orderings of a field 9 .

An ordering $v$ of a field $k$ is given by a binary relation $\leq_{v}$ such that for all $a, b, c \in k$ :

- $a \leq_{v} a$.

- If $a \leq_{v} b$ and $b \leq_{\nu} c$, then $a \leq_{v} c$.

- If $a \leq_{v} b$ and $b \leq_{v} a$, then $a=b$.

- Either $a \leq_{v} b$ or $b \leq_{v} a$.

- If $a \leq_{v} b$, then $a+c \leq_{v} b+c$.

- If $a \leq_{v} b$ and $0 \leq_{v} c$, then $c a \leq_{v} c b$.

A field $k$ which admits an ordering is necessarily of characteristic 0 . If $k$ is a field with an ordering $v$, an algebraic extension of the ordered field $(k, v)$ is an algebraic field extension $L$ of $k$ together with an ordering $v^{\prime}$ on $L$ such that $v^{\prime}$ restricted to $k$ is $v$. If $L$ is a finite field extension of $k$ of odd degree, then there is always an algebraic extension $\left(L, v^{\prime}\right)$ of $(k, v)$ [9, Chapter 3, Theorem 1.10].

A field $k$ is said to be formally real if -1 is not a sum of squares in $k$. A field $k$ is called a real closed field if it is a formally real field and no proper algebraic extension is formally real. There is a unique ordering $\square$ on a real closed field. This 
ordering is defined by the relation $a \leq b$ if and only if $b-a$ is a square in $k$. Further, if $k$ is a real closed field, then $k(\sqrt{-1})$ is algebraically closed [9, Theorem 2.3 (iii)].

If $L$ is a finite field extension of $k$, then $k_{v} \otimes L$ is isomorphic to a product of the form $\prod k_{v} \prod k_{v}(\sqrt{-1})$. Also, since $k_{v}(\sqrt{-1})$ is an algebraic closure for $k$ there is a natural inclusion $\operatorname{Gal}\left(\bar{k}, k_{v}\right) \subset \Gamma_{k}$ and thus a restriction map $H^{1}(k, G) \rightarrow$ $H^{1}\left(k_{v}, G\right)$.

\section{Main Result}

In the discussion which follows we will need the following lemmas.

Lemma 4.1. Let $k$ be a field and let $G$ be a reductive group over $k$. Fix an integer $n$ and a quasitrivial torus $T$ such that $G^{n} \times T$ admits a special covering

$$
1 \rightarrow \mu \rightarrow G_{0} \times S \rightarrow G^{n} \times T \rightarrow 1
$$

Then $G^{s c}$ satisfies a Hasse Principle over $k$ if and only if $G_{0}$ satisfies a Hasse principle over $k$.

Proof. Taking commutator subgroups we have a short exact sequence

$$
1 \rightarrow \tilde{\mu} \rightarrow\left[G_{0} \times S: G_{0} \times S\right] \rightarrow\left[G^{n} \times T: G^{n} \times T\right] \rightarrow 1 .
$$

Since $S$ and $T$ are tori, $\left[G_{0} \times S: G_{0} \times S\right] \cong\left[G_{0}: G_{0}\right]$ and $\left[G^{n} \times T: G^{n} \times\right.$ $T]=\left[G^{n}: G^{n}\right]$. That $G_{0}$ is semisimple gives $\left[G_{0}: G_{0}\right]=G_{0}$. It is clear that $\left[G^{n}: G^{n}\right]=[G: G]^{n}$, which in turn is $\left(G^{s s}\right)^{n}$ by the definition of $G^{s s}$. Therefore, we have the following short exact sequence:

$$
1 \rightarrow \tilde{\mu} \rightarrow G_{0} \rightarrow\left(G^{s s}\right)^{n} \rightarrow 1
$$

where $\tilde{\mu}$ is some finite group scheme. In particular, $G_{0}$ is a simply connected cover of $\left(G^{s s}\right)^{n}$. Since $\left(G^{s c}\right)^{n}$ is certainly a simply connected cover of $\left(G^{s s}\right)^{n}$, uniqueness of the simply connected cover of $\left(G^{s s}\right)^{n}$ gives $\left(G^{s c}\right)^{n} \cong G_{0}$. In particular, the simple factors of $G^{s c}$ are the same as the simple factors of $G_{0}$, and $G^{s c}$ satisfies the Hasse principle over $k$ if and only if $G_{0}$ satisfies the Hasse principle over $k$.

Lemma 4.2. Let $k$ be a real closed field and let $G$ be a reductive group over $k$ which admits a special covering

$$
1 \rightarrow \mu \rightarrow G_{0} \times S \rightarrow G \rightarrow 1
$$

Let $L$ be a finite étale $k$-algebra. Let $\delta$ be the first connecting map in Galois Cohomology and let $N_{L / k}$ denote the corestriction map $H^{1}(k \otimes L, \mu) \rightarrow H^{1}(k, \mu)$. Then

$$
N_{L / k}\left(\operatorname{im}\left(G(k \otimes L) \stackrel{\delta_{L}}{\longrightarrow} H^{1}(k \otimes L, \mu)\right)\right) \subset \operatorname{im}\left(G(k) \stackrel{\delta}{\longrightarrow} H^{1}(k, \mu)\right) .
$$

Proof. Since $k$ is real closed, there exist finite numbers $r$ and $s$ such that $k \otimes L$ is isomorphic to a product of $r$ copies of $k$ and $s$ copies of $k(\sqrt{-1})$. Thus

$$
H^{1}(k \otimes L, \mu) \cong \prod_{r \text { copies }} H^{1}(k, \mu) \prod_{s \text { copies }} H^{1}(k(\sqrt{-1}), \mu) .
$$

Since $k$ is real closed, $k(\sqrt{-1})$ is algebraically closed, $H^{1}(k(\sqrt{-1}), \mu)$ is trivial and $H^{1}(k \otimes L, \mu)$ is just a product of $r$ copies of $H^{1}(k, \mu)$. Therefore,

$$
N_{L / k}: H^{1}(k \otimes L, \mu) \rightarrow H^{1}(k, \mu)
$$


is just the product map

$$
\prod_{r \text { copies }} H^{1}(k, \mu) \rightarrow H^{1}(k, \mu) .
$$

That $k \otimes L$ is a product of $r$ copies of $k$ and $s$ copies of $k(\sqrt{-1})$ also gives that

$$
G(k \otimes L) \cong \prod_{r \text { copies }} G(k) \prod_{s \text { copies }} G(k(\sqrt{-1})) .
$$

Therefore, the connecting map

$$
\prod_{r \text { copies }} G(k) \prod_{s \text { copies }} G(k(\sqrt{-1})) \stackrel{\delta}{\longrightarrow} \prod_{r \text { copies }} H^{1}(k, \mu) \prod_{s \text { copies }} H^{1}(k(\sqrt{-1}), \mu)
$$

is just the product of the connecting maps

$$
G(k) \rightarrow H^{1}(k, \mu)
$$

and

$$
G(k(\sqrt{-1})) \rightarrow H^{1}(k(\sqrt{-1}), \mu),
$$

the latter of which is necessarily the trivial map.

So choose

$$
\left(x_{1}, \ldots, x_{r}, y_{1}, \ldots, y_{s}\right) \in G(k \otimes L) .
$$

Then

$$
\begin{aligned}
N_{L / k}\left(\delta\left(x_{1}, \ldots, x_{r}, y_{1}, \ldots, y_{s}\right)\right) & =N_{L / k}\left(\delta\left(x_{1}\right), \ldots, \delta\left(x_{r}\right), \delta\left(y_{1}\right), \ldots, \delta\left(y_{s}\right)\right) \\
& =\delta\left(x_{1}\right) \cdots \delta\left(x_{r}\right) \\
& =\delta\left(x_{1} \cdots x_{r}\right) .
\end{aligned}
$$

Since the $x_{i}$ were chosen to be in $G(k)$ for all $i$, then $x_{1} \cdots x_{r} \in G(k)$ and the desired result holds.

Lemma 4.3. Let $G$ be a reductive group and $L$ be a finite field extension of $k$ of odd degree. The kernel of the canonical map $H^{1}(k, G) \rightarrow H^{1}(L, G)$ is contained in the kernel of the canonical map $H^{1}(k, G) \rightarrow \prod_{v \in \Omega} H^{1}\left(k_{v}, G\right)$.

Proof. By [9, Chapter 3, Theorem 1.10] each ordering $v$ of $k$ extends to an ordering $w$ of $L$; in particular, each real closure $k_{v}$ is $L_{w}$ for some ordering $w$ on $L$. Since the natural map $H^{1}(k, G) \rightarrow H^{1}\left(L_{w}, G\right)$ factors through the canonical map $H^{1}(k, G) \rightarrow$ $H^{1}(L, G)$, the desired result is immediate.

We now return to the result which is the main goal of this paper.

Theorem 4.4. Let $k$ be a perfect field of virtual cohomological dimension $\leq 2$ and let $G$ be a connected linear algebraic group over $k$. Let $\left\{L_{i}\right\}_{1 \leq i \leq m}$ be a set of finite field extensions of $k$ such that the greatest common divisor of the degrees of the extensions $\left[L_{i}: k\right]$ is 1 . If $G^{s c}$ satisfies a Hasse principle over $k$, then the canonical map

$$
H^{1}(k, G) \rightarrow \prod_{i=1}^{m} H^{1}\left(L_{i}, G\right)
$$

has trivial kernel. 
Proof. By definition of the groups involved, the following sequence is exact:

$$
1 \rightarrow G^{u} \rightarrow G \rightarrow G^{\mathrm{red}} \rightarrow 1 .
$$

Since $G^{u}$ is unipotent, $H^{i}\left(k, G^{u}\right)$ is trivial for $i \geq 1$ and (4.4.1) induces the long exact sequence in Galois Cohomology

$$
1 \rightarrow H^{1}(k, G) \rightarrow H^{1}\left(k, G^{\mathrm{red}}\right) \rightarrow 1,
$$

which gives that $H^{1}(k, G) \cong H^{1}\left(k, G^{\text {red }}\right)$. Thus to prove Theorem 4.4 it is sufficient to consider the case where $G$ is a reductive group. Then fix an integer $n$ and quasitrivial torus $T$ such that $G^{n} \times T$ admits a special covering

$$
1 \rightarrow \mu \rightarrow G_{0} \times S \rightarrow G^{n} \times T \rightarrow 1
$$

By functoriality, $H^{1}\left(k, G^{n} \times T\right) \cong H^{1}(k, G)^{n} \times H^{1}(k, T)$ and since $T$ is quasitrivial, $H^{1}(k, T)=1$. It follows that our result holds for $G$ if and only if it holds for $G^{n} \times T$. Replacing $G$ by $G=G^{n} \times T$ we assume that $G$ admits a special covering

$$
1 \rightarrow \mu \rightarrow G_{0} \times S \rightarrow G \rightarrow 1 .
$$

If $k$ is a field of positive characteristic, $\operatorname{cd}(d)=\operatorname{vcd}(k)=2$. Since $k$ has no orderings and by hypothesis $G^{s c}$ satisfies a Hasse principle over $k$, then $H^{1}\left(k, G^{s c}\right)=$ $\{1\}$. In particular $H^{1}\left(k, G_{0}\right)=\{1\}$ and the special covering of $G$ above induces the following commutative diagram with exact rows:

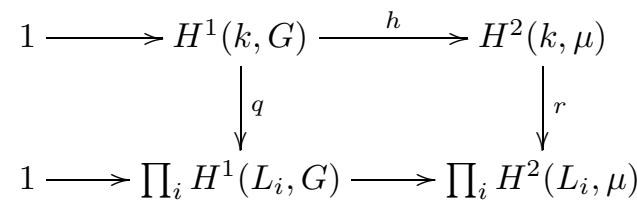

Choose $\lambda \in \operatorname{ker}(q)$. By commutativity of the diagram, $h(\lambda) \in \operatorname{ker}(r)$. A restrictioncorestriction argument gives that $r$ has trivial kernel. Thus $h(\lambda)=$ point. Then by exactness of the top row of the diagram, $\lambda=$ point. (Cf. 22 for the case that $k$ is a "good" field of cohomological dimension 2.)

Therefore, we may assume that the characteristic of $k$ is zero. Fix an index $i$. The special covering of $G$ above induces the following commutative diagram with exact rows where the vertical maps are the restriction maps:

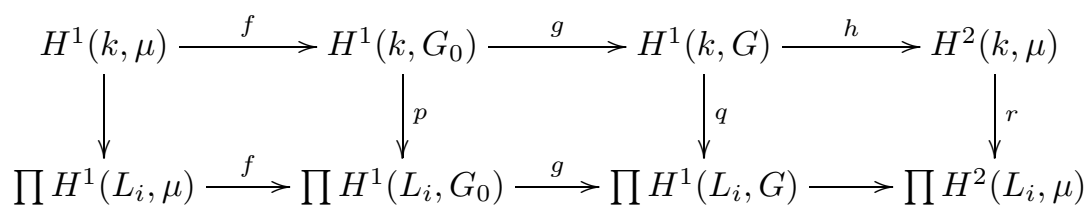

Let $\lambda$ be in $\operatorname{ker}(q)$. Taking cores o res we find that $r$ has trivial kernel and thus by commutativity of (4.4.3), $\lambda$ is in $\operatorname{ker}(h)$. By exactness of the top row, we choose $\lambda^{\prime} \in H^{1}\left(k, G_{0}\right)$ such that $g\left(\lambda^{\prime}\right)=\lambda$. Write $p\left(\lambda^{\prime}\right)=\left(\lambda_{L_{i}}^{\prime}\right)$. Since $g\left(\lambda_{L_{i}}^{\prime}\right)=$ point, by exactness of the bottom row of (4.4.3) choose $\eta_{L_{i}} \in H^{1}\left(L_{i}, \mu\right)$ such that $f\left(\eta_{L_{i}}\right)=$ $\lambda_{L_{i}}^{\prime}$ 
For each ordering $v$ of $k$, the special covering of $G$ above also induces the following commutative diagram with exact rows:

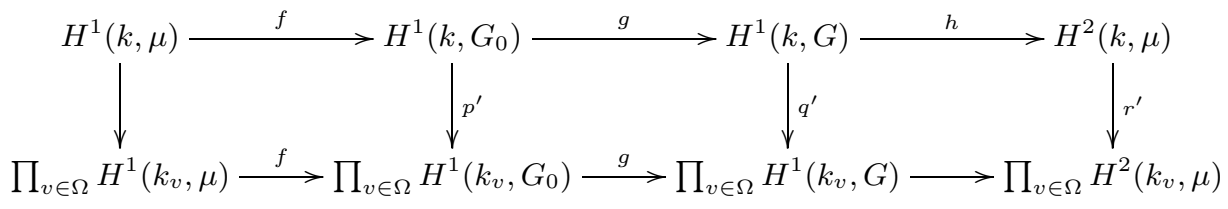

By Lemma 4.3, $\lambda$ is in the kernel of $q^{\prime}$. Thus by commutativity of 4.4.4), $\left(\lambda_{v}^{\prime}\right)=$ $p^{\prime}\left(\lambda^{\prime}\right)$ is in $\operatorname{ker}(g)$. Then by exactness of the bottom row of (4.4.4) choose $\alpha_{v} \in$ $H^{1}\left(k_{v}, \mu\right)$ such that $f\left(\alpha_{v}\right)=\lambda_{v}^{\prime}$. Let $\left(\alpha_{v}\right)_{L_{i}}$ denote the image of $\alpha_{v}$ under the canonical map $H^{1}\left(k_{v}, \mu\right) \rightarrow H^{1}\left(k_{v} \otimes L_{i}, \mu\right)$. Let $\left(\eta_{L_{i}}\right)_{v}$ denote the image of $\eta_{L_{i}}$ under the canonical map $H^{1}\left(L_{i}, \mu\right) \rightarrow H^{1}\left(k_{v} \otimes L_{i}, \mu\right)$.

By choice of $\alpha_{v}$ and $\eta_{L_{i}}, f\left(\left(\alpha_{v}\right)_{L_{i}}\right)=\left(\lambda_{v}^{\prime}\right)_{L_{i}}=\left(\lambda_{L_{i}}^{\prime}\right)_{v}=f\left(\left(\eta_{L_{i}}\right)_{v}\right)$. In particular, $f\left(\left(\alpha_{v}\right)_{L_{i}}\left(\eta_{L_{i}}\right)_{v}^{-1}\right)$ is the point in $H^{1}\left(k_{v} \otimes L_{i}, G_{0}\right)$. We have a commutative diagram

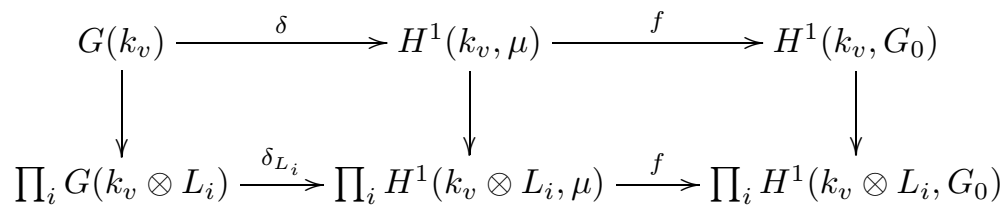

Exactness of the bottom row of (4.4.5) gives that $\left(\alpha_{v}\right)_{L_{i}}\left(\eta_{L_{i}}\right)_{v}^{-1}$ is in the image of $\delta_{L_{i}}$. Choose $m_{i}$ such that $\sum m_{i}\left[L_{i}: k\right]=1$. Since $\delta_{L_{i}}$ is multiplicative, it follows that for each index $i,\left(\alpha_{v}\right)_{L_{i}}^{m_{i}}\left(\left(\eta_{L_{i}}\right)_{v}^{-1}\right)^{m_{i}}$ is in the image of $\delta_{L_{i}}$.

By Lemma 4.2 above, there exists $\gamma_{v}$ in $G\left(k_{v}\right)$ such that

$$
\delta\left(\gamma_{v}\right)=\prod_{i} N_{L_{i} / k}\left(\left(\alpha_{v}\right)_{L_{i}}^{m_{i}}\left(\left(\eta_{L_{i}}\right)_{v}^{-1}\right)^{m_{i}}\right)
$$

Since by restriction-corestriction $N_{L_{i} / k}\left(\left(\alpha_{v}\right)_{L_{i}}^{m_{i}}\right)=\alpha_{v}^{m_{i}\left[L_{i}: k\right]}$, it follows that

$$
\begin{aligned}
\delta\left(\gamma_{v}\right) & =\prod_{i} N_{L_{i} / k}\left(\left(\alpha_{v}\right)_{L_{i}}^{m_{i}}\left(\left(\eta_{L_{i}}\right)_{v}^{-1}\right)^{m_{i}}\right) \\
& =\alpha_{v}^{\sum_{i} m_{i}\left[L_{i}: k\right]} \prod_{i}\left(N_{L_{i} / k}\left(\eta_{L_{i}}\right)_{v}^{-1}\right)^{m_{i}} \\
& =\alpha_{v} \prod_{i}\left(N_{L_{i} / k}\left(\eta_{L_{i}}\right)_{v}^{-1}\right)^{m_{i}} .
\end{aligned}
$$

In turn

$$
\delta\left(\gamma_{v}\right) \prod_{i}\left(N_{L_{i} / k}\left(\eta_{L_{i}}\right)_{v}\right)^{m_{i}}=\alpha_{v} .
$$

Since $f$ is well-defined on the cosets of $G\left(k_{v}\right)$ in $H^{1}\left(k_{v}, \mu\right)$ [7] and the top row of (4.4.5) is exact, it follows that

$$
f\left(\prod_{i}\left(N_{L_{i} / k}\left(\eta_{L_{i}}\right)_{v}\right)^{m_{i}}\right)=f\left(\alpha_{v}\right) .
$$

By choice of $\alpha_{v}$ the latter is $\lambda_{v}^{\prime}$. Since $G^{s c}$ satisfies a Hasse principle over $k$, Lemma 4.1 gives that $G_{0}$ satisfies a Hasse principle over $k$. In particular, the map 
$H^{1}\left(k, G_{0}\right) \rightarrow \prod_{v} H^{1}\left(k_{v}, G_{0}\right)$ is injective, and since $f\left(\prod_{i}\left(N_{L_{i} / k}\left(\eta_{L_{i}}\right)^{m_{i}}\right)\right)_{v}=\lambda_{v}^{\prime}$ for all $v$, then

$$
f\left(\prod_{i}\left(N_{L_{i} / k}\left(\eta_{L_{i}}\right)\right)^{m_{i}}\right)=\lambda^{\prime} .
$$

Taking $g$ as in (4.4.3) above,

$$
g\left(f\left(\prod_{i}\left(N_{L_{i} / k}\left(\eta_{L_{i}}\right)\right)^{m_{i}}\right)\right)=g\left(\lambda^{\prime}\right) .
$$

Then by exactness of the top row of (4.4.3), $\lambda=g\left(\lambda^{\prime}\right)=$ point.

Applying [1, Theorem 10.1] and a Serre twist we obtain the following corollary:

Corollary 4.5. Let $k$ be a perfect field of virtual cohomological dimension $\leq 2$. Let $\left\{L_{i}\right\}_{1 \leq i \leq m}$ be a set of finite field extensions of $k$ such that the greatest common divisor of the degrees of the extensions $\left[L_{i}: k\right]$ is 1 . Let $G$ be a connected linear algebraic group over $k$. If the simple factors of $G^{\text {sc }}$ are of classical type, type $F_{4}$ or type $G_{2}$, then the canonical map

$$
H^{1}(k, G) \rightarrow \prod_{i=1}^{m} H^{1}\left(L_{i}, G\right)
$$

is injective.

\section{REFERENCES}

1. E. Bayer-Fluckiger and R. Parimala, Classical groups and the Hasse principle, Ann. of Math. (2) 147 (1998), no. 3, 651-693. MR.1637659 (99g:11055)

2. M. Borovoi, J.-L. Colliot-Thélène, and A. N. Skorobogatov, The elementary obstruction and homogeneous spaces, Duke Math. J. 141 (2008), no. 2, 321-364. MR2376817 (2009f:14040)

3. V. I. Chernousov, The Hasse principle for groups of type $E_{8}$, Dokl. Akad. Nauk SSSR 306 (1989), no. 5, 1059-1063. MR.1014762 (90m:11055)

4. Günter Harder, Über die Galoiskohomologie halbeinfacher Matrizengruppen. II, Math. Z. 92 (1966), 396-415. MR0202918 (34:2777)

5. Martin Kneser, Hasse principle for $H^{1}$ of simply connected groups, Proc. Sympos. Pure Math., Amer. Math. Soc., 1965, pp. 159-163. MR0220736 (36:3788)

6. Max-Albert Knus, Alexander Merkurjev, Markus Rost, and Jean-Pierre Tignol, The book of involutions, American Mathematical Society Colloquium Publications, vol. 44, American Mathematical Society, Providence, RI, 1998, with a preface in French by J. Tits. MR $\overline{1632779}$ (2000a:16031)

7. A. S. Merkur'ev, The norm principle for algebraic groups, Algebra i Analiz 7 (1995), no. 2, 77-105. MR:1347513 (96k:20088)

8. J.-J. Sansuc, Groupe de Brauer et arithmétique des groupes algébriques linéaires sur un corps de nombres, J. Reine Angew. Math. 327 (1981), 12-80. MR631309 (83d:12010)

9. Winfried Scharlau, Quadratic and Hermitian forms, Grundlehren der Mathematischen Wissenschaften [Fundamental Principles of Mathematical Sciences], vol. 270, Springer-Verlag, Berlin, 1985. MR 770063 (86k:11022)

10. Jean-Pierre Serre, Galois cohomology, English ed., Springer Monographs in Mathematics, Springer-Verlag, Berlin, 2002, translated from the French by Patrick Ion and revised by the author. MR1867431 (2002i:12004)

Department of Mathematics and Computer Science, Emory University, Atlanta, Georgia 30322

E-mail address: jablack@emory.edu 